\title{
Prevention of LPS- induced Acute Respiratory Distress Syndrome in Sheep by Bone Marrow-Derived Mesenchymal Stem/Stromal Cells
}

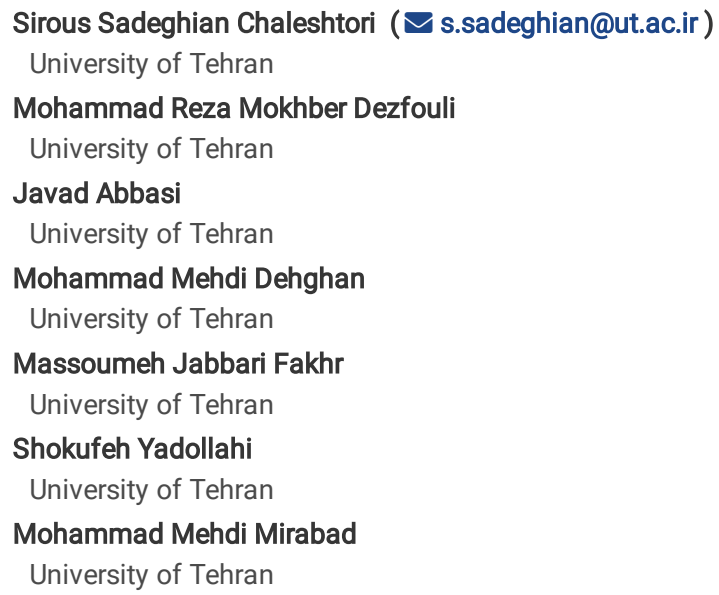




\section{Abstract}

In this study, 10 male Shall sheep were used in two groups and bone marrow samples were collected and BM-MSCs isolated. Then experimental model of ARDS was induced by intrapulmonary injection of LPS to dose of $400 \mu \mathrm{g} / \mathrm{kg}$. Twenty-four hours after LPS injection, $5 \times 10^{7}$ cells of BM-MSCs were autologous transferred in the group of treatment and $1 \mathrm{ml}$ PBS was infused in the group of control as intrapulmonary. Then, the symptoms of clinical, complete blood count, analysis of arterial blood gases and the concentrations of IL6,IL10,TNF-a,total protein, Ig M and albumin BAL were determined before and at times of $3,6,12,24,48,72$, and 168 after transplantation/infusion. The results of the investigations 24 hours post-LPS injection(time 0 ) indicated the occurrence of acute inflammation which confirmed ARDS model. These changes included increase in RR, HR and RT, decrease in PO2 and SatO2 and increase in PCO2, WBC, neutrophils, macrophages, total protein, IL6, IL10, TNF-a, Ig M and albumin. But the stem/stromal cells transplantation reduced the severity of clinical signs induced by LPS, caused significant increase in $\mathrm{PO}_{2}, \mathrm{SatO}_{2}$ and IL-10 and significant decrease in $\mathrm{PCO}_{2}$, the total protein, TNF-a, IL-6, Ig M, albumin, WBCs, neutrophils and macrophages at different times of sampling both in compared with before transplantation(time 0) and in compared with the group of control. While in the control group, inflammation continued until the end of the study. These results showed that BM-MSCs are able to reduce inflammation and have an important role in reconstruction of the damaged lung.

\section{Introduction}

Acute respiratory distress syndrome (ARDS) is a clinical concept that is defined by acute hypoxemic failure, bilateral widespread capillary leakage and low lung compliance ${ }^{1}$. ARDS develops with the release of several proinflammatory proteins, and lipids and permeation of neutrophils into alveoli, is then followed by protein-rich pulmonary edema, surfactant inactivation and disruption of alveolar-capillary barrier ${ }^{2}$. ARDS is caused by response to multiple predisposing factors, including pulmonary and extrapulmonary factors and infectious and noninfectious agents, of which sepsis is the most important and accounts for $75 \%$ of these ${ }^{3,4}$. In addition, the heterogeneity, complexity and variety of the ARDS mechanism combined with the failure of current therapies for ARDS and the mortality rate $36-44 \%{ }^{5}$, require novel therapeutic interventions in ARDS that can target different mechanisms of injury and facilitate the lung repair.

Cell-based therapies with mesenchymal stem/stromal cells (MSCs) might be appropriate for this purpose. Among all of the MSCs sources, bone marrow has the most experimental data to support their potential treatment. MSCs apply their favorable effects by release of paracrine factors, antimicrobial factors and up-regulating phagocytosis ${ }^{6}$.

Our results in preclinical model in prior publication provided strong details on stem/stromal cell based therapy in this field and demonstrated the resolving effects of this approach in clinical usage ${ }^{7}$. To achieve a therapeutic resolution and translatable model for use in human clinical trial, hypotheses must be confirmed in animal models. Without living systems therapeutic theories would not be valid. Animal studies are categorized, so we first developed BM-MSC therapy on ARDS model of rabbit ${ }^{7}$ and in the present study, we used ARDS model of sheep. The sheep model has similar anatomy, physiology and pathology to human. Large-animal ARDS models have greater clinical translational potential due to the ability for gas exchange performance, pulmonary hemodynamics, and ventilation mismatch ${ }^{8,9}$.

One of the validated clinical models of ARDS is induction with LPS in which LPS stimulate a TL4 mediated inflammatory response and serves as a good model of host innate immune response ${ }^{10}$. Clinical appearances develop, usually within 24 to $48 \mathrm{~h}$ after pulmonary artery pressure increases. An elevation in pulmonary vascular resistance and cardiac output leads to pulmonary hypertension. A decrease in mean systemic arterial pressure due to a decrease in systemic vascular resistance may also occur in ARDS patients as the systemic inflammatory response evolves.

Although species susceptibility to LPS is very different, induction with low dose LPS leads to inflammation of lung in sheep ${ }^{8}$, but cell therapy can modulate these changes in all species. Therefore, the present study was designed to evaluate the effects of therapeutic intrapulmonary autologous transplantation of BM-MSCs in ARDS induced by E. coli LPS in sheep.

\section{Materials And Methods}

\section{Animal Care}

Ten male Shall sheep with mean weight $35 \pm 4 \mathrm{~kg}$ were selected and their health was confirmed with clinical and paraclinical examinations and they were randomly divided into two groups of treatment (BM-MSCs recipient group, $n=5$ ) and control (PBS recipient group, $n=5$ ). Then, all sheep were kept in a place at the Institute of Biomedical Research, University of Tehran in ventilated conditions and appropriate light and temperature. The rations of sheep during the study period included alfalfa and barley. All protocols of animals and experiments were checked and appropriated by the Animal Research Ethical Committee of University of Tehran (Tehran, Iran).

\section{Sampling from bone marrow (BM) and isolation and culture of MSCs}

First, the sheep of the treatment group were off feed for $12 \mathrm{~h}$ and then anaesthetized with $35 \mathrm{mg} / \mathrm{kg}$ ketamine $10 \%$ (Alfasan-Holland), and $5 \mathrm{mg} / \mathrm{kg}$ xylazine $2 \%$ (Interchemie-Holland) as IM. After that, BM samples (about $5 \mathrm{ml}$ ) were collected from anaesthetized animal illiac crest in aseptic condition. Then, under GMP conditions, BM-MSCs were isolated with density gradient method, ultimately the cell precipitation were transfered into $25 \mathrm{~cm} 2$ flasks with DMEM- high glucose (Gibco), 20\% FBS (Gibco) and $100 \mathrm{U} / \mathrm{ml}$ penicillin/streptomycin (Biowest, France) and placed at $37^{\circ} \mathrm{C}$ in a humid atmosphere of $5 \% \mathrm{CO} 2$ (Memert, USA). The medium was replaced every 3-4 days. When confluency of the cells reach more than $80 \%$, the monolayer cells were trypsinized with $0.25 \%$ trypsinEDTA (Gibco, USA) and passaged.

\section{Characterization of BM-MSCs}


The BM-MSCs at passage three were harvested and labeled with PE-conjugated antibodies against CD45 (Biolegend, Inc), CD29, CD31 and CD44 (Abcam, Inc.), and then the cells were analyzed using flow cytometry (BD Bioscience, USA) and the Flowjo program version 7.6.1. In addition, to check multi-lineage differentiation ability of BM-MSCs, they were separately treated with osteogenic differentiation medium for 21 days and adipogenic differentiation medium for 14 days. Then the cells were fixed with Paraformaldehyde $4 \%$ for 20 min at room temperature and were respectively stained with Alizarin Red (Bioidea-Iran) for $10 \mathrm{~min}$ and Oil Red O (Bioidea-Iran) for $15 \mathrm{~min}$ at room temperature. After that, cells were washed with distilled water to remove excess stain and were observed using the Olympus IX71 inverted microscope.

\section{ARDS modeling in sheep}

\section{The dose calculation of $E$. coli LPS and ARDS establishment}

First, one vial of LPS from E. coli 055: B5, $10 \mathrm{mg}$ (Sigma-Aldrich) was dissolved in $10 \mathrm{ml}$ of sterile PBS and divided into sterilized microtubes, and were stored in freezer at -80 खC. After this, five Shall sheep weighing 30-35 kg were selected and anesthetized and intubated, and doses of 50, 100, 200, 400 and $800 \mu \mathrm{g} / \mathrm{kg}$ diluted in PBS heated to $37^{\circ} \mathrm{C}$ were intrapulmonary prescribed. Then, the sheep were clinically and paraclinically investigated and finally, according to the findings, the dose of $400 \mu \mathrm{g} / \mathrm{kg}$ was confirmed for inflammation in this study.

In the next stage, all sheep both in the treatment group and in the control group, were anesthetized and intubated and ARDS experimental model was induced by LPS at $400 \mu \mathrm{g} / \mathrm{kg}$. The findings of clinical signs, radiography, blood and BAL profiles and blood gases analysis were recorded before and after administration of LPS.

\section{Autologous transplantation of BM-MSCs}

In the treatment group, $24 \mathrm{~h}$ after induction of ARDS, first, viability and number of BM-MSCs were assessed by trypan blue staining. Then, under general anesthesia, sheep were sternaly placed, trachea was cannulated. Then, autologous transplantation of $5 \times 10^{7}$ BM-MSCs were intrapulmonary done. Also, in the control group, $1 \mathrm{ml}$ PBS was intrapulmonary infused only, one day after induction of ARDS.

\section{Clinical and laboratory investigations}

The sheep both in the treatment group (ARDS+BM-MSCs) and in the control group (ARDS+PBS) were monitored by clinical examination, evaluation of blood and BAL samples consisted of blood gases analysis, hematologic analysis and measurement of cytokines before establishment of ARDS (time -24), time of BM-MSCs transplantation/PBS infusion (times 0 ) and then for $3,6,12,24,48,72,168 \mathrm{~h}$ after BM-MSCs transplantation/PBS infusion.

\section{Clinical examination}

The clinical symptoms of RR, HR, RT, breathing sound, cough, nasal discharge, mucosal membrane, appetite and physical condition were determined and documented based on clinical scores for each sheep. The scoring is based on examination of clinical benchmark according to scientific texts and articles ${ }^{11,12}$ that were individually specified for each sheep (Table 1).

\begin{tabular}{|c|c|c|c|c|c|}
\hline Score & 0 & 1 & 2 & 3 & 4 \\
\hline $\begin{array}{c}\text { Signs } \\
\text { Breathing sound }\end{array}$ & Normal & - & - & $\begin{array}{c}\text { Abnormal } \\
\text { (Crackle-Wheeze) }\end{array}$ & - \\
\hline $\begin{array}{c}\text { Cough } \\
\text { Nasal discharge }\end{array}$ & $\begin{array}{l}\text { Absent } \\
\text { Absent }\end{array}$ & $\begin{array}{l}\text { A single cough } \\
\text { Serous }\end{array}$ & $\begin{array}{l}\text { Involuntary, repetitive coughs } \\
\text { mucus }\end{array}$ & $\begin{array}{l}\text { Repetitive coughs } \\
\text { Muco-purulent }\end{array}$ & Purulent \\
\hline Mucosal membrane & Normal & Mild hyperemia & Severe hyperemia & Cyanotic & - \\
\hline Appetite & Normal & Decrease $(<50 \%)$ & Decrease $(\geq 50 \%)$ & Anorexie & - \\
\hline Physical condition & $\begin{array}{c}\text { Normal } \\
\text { (alert) }\end{array}$ & Dull (slowly response) & Depressed (acutely slow response) & Severe depression (recumbent) & - \\
\hline
\end{tabular}

\section{Sampling and assessment of blood and BAL samples}

Blood samples were collected from the ear artery and jugular vein into syringes containing the anticoagulation. Arterial samples containing heparin were used for blood gases analyses by Blood Gas Analyzers (OPTI CCA-TS) and the parameters included pH, PO2, PCO2, HCO3, TCO2, SatO2 and anion gap and electrolytes such as $\mathrm{Na}^{+}, \mathrm{K}^{+}$, and $\mathrm{Cl}^{-}$were measured.

Venous samples containing EDTA were distributed in two microtubes. One microtube was immediately used for haematologic parameters analysis, and the other was centrifuged at $800 \mathrm{~g}$ at $4{ }^{\circ} \mathrm{C}$ for $20 \mathrm{~min}$. Then, plasma was separated and frozen at $-80{ }^{\circ} \mathrm{C}$ for measurement of cytokines. The pro and antiinflammatory cytokines concentrations including TNF-a, IL-6, and IL-10 were measured with commercially available ELISA kits (Eastbiopharm-USA) following the manufacturer's protocols. 
Also, after general anaesthetizing with ketamine and xylazine, sheep were sternaly positioned and lung wash was performed with PBS via endotracheal tube. $10 \mathrm{ml}$ sterile normal saline (room temperature) was instilled into lung and immediately aspirated and samples of BAL were collected. Then, samples were centrifuged at $400 \mathrm{~g}$ at $4{ }^{\circ} \mathrm{C}$ for $10 \mathrm{~min}$ and the supernatants were placed at fireezer at $-80{ }^{\circ} \mathrm{C}$ for measurement of cytokines and supernatants from pellets were used for complete cell counts. The pro and anti-inflammatory cytokines concentrations including TNF-a, IL-6, and IL-10 and concentrations of Ig M, albumin and total protein to evaluate the pulmonary vascular permeability were measured with commercially available ELISA kits (Eastbiopharm-USA) following the manufacturer's protocols. In addition, smears were prepared from pellets and stained by Giemsa and total cell count, neutrophils and macrophages were counted.

\section{Statistical analysis}

The results were analyzed statistically by SPSS software (version 24). Data were assessed by the independent samples t-test, repeated measure, MannWhitney $\mathrm{U}$ and Friedman tests at the significance level $\mathrm{p}<0.05$.

\section{Results}

\section{Isolation, culture and characterizations of BM-MSCs in sheep}

Cells that are isolated from the BM were spherical early. Because of the MSCs adhesion, gradually with changing the cell medium and passage, the purity of the MSCs increased and other cells were removed. BM-MSCs were initially appendages, and then had a fibroblastic-like appearance and spindle shape. After some days, $80-90 \%$ of the culture flask was covered with spindle cells. In the present study, the cells had broad and stretched morphology and vortex patterns after three consecutive passages.

Differentiation of BM-MSCs to lineages of adipogenic and osteogenic revealed, MSCs preserved their ability to form adipocytes and osteoblasts in medium of differentiation and proved potential multipotent MSCs. Also, the findings Flow cytometry showed that BM-MSCs expressed cell surface markers of CD29 and CD44, 91\% and 89\%, respectively, but did not express markers of CD31 (marker of endothelial cells) and CD45 (marker of hematopoietic cells) (Figure 1). These results confirmed cultured cells were MSCs.

\section{The lethal dose of $E$. coli LPS and ARDS model confirmation}

Doses of 50,100 and $200 \mu \mathrm{g} / \mathrm{kg}$ did not cause significant pulmonary inflammation but the $400 \mu \mathrm{g} / \mathrm{kg}$ dose caused acute pulmonary inflammation, and the dose of $800 \mu \mathrm{g} / \mathrm{kg}$ caused death in sheep. Thus, the $400 \mu \mathrm{g} / \mathrm{kg}$ dose was used for establishment of ARDS experimental model. It should also be mentioned that individual sensitivities in sheep were not observed in the $400 \mu \mathrm{g} / \mathrm{kg}$ dose.

The results of the investigations one day after injection of LPS compared to the normal state indicated the occurrence of acute inflammation. Clinical changes included respiratory abnormal sounds (crackle and wheeze), difficulty breathing, cough, increase in RR ( $p=0.018), H R(p=.014), R T(p=0.001)$, mucosal hyperemia, abnormal discharge from the nose, decreased appetite and abnormal physical condition. In addition, hematological changes included WBC $(p=0.015)$ and segmented neutrophils $(p=0.03), P O 2(p=0.019), P C O 2(p=0.04)$ and measurement of inflammatory factors in BAL demonstrated changes in total protein ( $p=0.045)$, IL6/total protein ( $p=0.02)$, IL10/total protein ( $p=0.02)$, TNF-a/total protein $(p=0.002)$, Ig M/total protein ( $p=0.000)$ and albumin/total protein $(\mathrm{p}=0.031)$. All these results confirmed the acute pulmonary involvement and ARDS model.

\section{Results of clinical and laboratory after BM-MSCs transplantation in ARDS sheep}

\section{MSCs: recovery of clinical symptoms}

After the cell therapy in the treatment group (ARDS+BM-MSCs), a decrease in HR observed since time 6 hours next. So, 24, 48 and $72 \mathrm{~h}$ post-transplantation of BM-MSCs compared with before transplantation (time 0 ) ( $p$ value was $0.045,0.028$, and 0.025 , respectively) and compared with similar times in the group of control (ARDS+PBS) ( $p$ value was $0.013,0.034$, and 0.038 , respectively) were significant.

RR reduction was significant in the cell receiver group at times of $12(p=0.033), 24(p=0.029), 48(p=0.040), 72(p=0.035)$ and 168 ( $p=0.022)$ compared with time 0 and at $24(p=0.045), 48(p=0.026), 72(p=0.042)$ and 168 hours $(p=0.031)$ compared with the PBS receiver group.

RT decline was begun after stem cells transplantation and at the end of the study, it returned to the base level, so that was significant at $12,24,48,72$ and 168 times compared with time 0 ( $p$ value was $0.030,0.016,0.040,0.018$ and 0.002 , respectively). In addition, a significant difference seen after the cell therapy in comparison with the control group at hours of $24,48,72$ and 168 ( $p$ value was $0.044,0.019,0.011$ and 0.021 , respectively) (Table 2). 


\begin{tabular}{|c|c|c|c|c|c|c|c|c|c|c|}
\hline \multirow{2}{*}{$\begin{array}{c}\text { Clinical } \\
\text { Symptoms }\end{array}$} & (Time (h & -24 & 0 & 3 & 6 & 12 & 24 & 48 & 72 & 168 \\
\hline & Group & & & & & & & & & \\
\hline \multirow[t]{2}{*}{$\mathrm{HR} / \mathrm{min}$} & Treatment & $* 4.3 \pm 86.40$ & $3.28 \pm 109.40$ & $11.83 \pm 110.0$ & $12.13 \pm 104.20$ & $11.21 \pm 100.80$ & $\begin{array}{c}* 1.58 \pm 95.00 \\
\#\end{array}$ & $\begin{array}{c}2.50 \pm 92.60 \\
\# *\end{array}$ & $\begin{array}{c}4.03 \pm 92.60 \\
\# *\end{array}$ & $6.34 \pm 91.60$ \\
\hline & Control & $* 2.16 \pm 85.80$ & $4.60 \pm 107.80$ & $5.58 \pm 110.20$ & $6.22 \pm 110.40$ & $5.89 \pm 107.20$ & $40.60 \pm 107.80$ & $5.31 \pm 106.40$ & $9.54 \pm 102.80$ & $4.47 \pm 104.00$ \\
\hline \multirow[t]{2}{*}{$\mathrm{RR} / \min$} & Treatment & $* 1.81 \pm 22.60$ & $5.68 \pm 42.60$ & $5.09 \pm 41.0$ & $9.09 \pm 38.20$ & $* 4.84 \pm 36.0$ & $\begin{array}{c}* 4.43 \pm 35.20 \\
\#\end{array}$ & $\begin{array}{c}4.20 \pm 31.20 \\
\# *\end{array}$ & $\begin{array}{c}3.93 \pm 29.00 \\
\# *\end{array}$ & $\begin{array}{c}* 3.08 \pm 25.00 \\
\#\end{array}$ \\
\hline & Control & $* 2.16 \pm 22.20$ & $3.27 \pm 41.20$ & $3.28 \pm 4.60$ & $3.78 \pm 41.40$ & $2.30 \pm 42.40$ & $7.52 \pm 41.80$ & $1.51 \pm 39.40$ & $37 \pm 1.58$ & $5.71 \pm 34.801$ \\
\hline \multirow[t]{2}{*}{ RT } & Treatment & $* \pm 039.16 .2$ & \pm 040.10 .14 & \pm 040.06 .16 & \pm 039.88 .25 & $* \pm 039.64 .24$ & $\begin{array}{c} \pm 039.48 .04 \\
\# *\end{array}$ & $\begin{array}{c} \pm 039.38 .20 \\
\# *\end{array}$ & $\begin{array}{c} \pm 039.28 .26 \\
\# *\end{array}$ & $\begin{array}{c} \pm 039.22 .22 \\
\# *\end{array}$ \\
\hline & Control & $* \pm 0.1539 .10$ & \pm 040.06 .20 & \pm 040.08 .25 & \pm 040.14 .16 & \pm 040.08 .16 & \pm 040.02 .51 & \pm 040.08 .10 & \pm 039.94 .08 & \pm 039.80 .07 \\
\hline
\end{tabular}

Twenty-four hours after transplantation of MSCs, changes in pulmonary sounds (including wheeze, crackle) decreased gradually so that these changes were significant compared with the PBS receiver group at $72 \mathrm{~h}(\mathrm{p}=0.014)$ and $168 \mathrm{~h}(\mathrm{p}=0.014)$. Improvement in the appetite of sheep in the treatment group began since time $6 \mathrm{~h}$ next, so at time of $72 \mathrm{~h}$ the appetite was completely normal. Comparison between the two groups revealed a significant difference at hours of $12(p=0.015), 24(p=0.017), 48(p=0.014), 72(p=0.007)$, and $168(p=0.005)$ post-transplantation.

After inflammation, nasal discharge was seen as unilateral or bilateral that was sometimes accompanied by changes of color in both groups. But one week after BM-MSCs transplantation there was no secretion of the nostrils and in comparison with the group of control demonstrated a significant difference at 24 , 48,72 and 168 times ( $p$ value was $0.031,0.007,0.008$ and 0.006 , respectively). The cough results also indicated that there was a decrease in the number of coughs in the treatment group since time of $48 \mathrm{~h}$ until there was no cough at $168 \mathrm{~h}$ even with stimulation of reflex. Statistical comparison also showed that in the cell receiver group, at times of $24(p=0.031), 48(p=0.014), 72(p=0.014)$ and 168 hours $(p=0.006)$ cough was significantly different compared to the control group.

In the treatment group, the severity of mucosal hyperemia reduced at $48 \mathrm{~h}$ after transplantation and it returned to the state of pre-inflammation (time of - $24 \mathrm{~h}$ ) at $72 \mathrm{~h}$ and also, demonstrated a significant difference at hours of $24,48,72$ and 168 compared to the control group ( $p$ value was $0.014,0.031,0.021$ and 0.005 , respectively). In addition, the cell therapy caused physical condition and consciousness of the sheep to return to normal at $72 \mathrm{~h}$ after transplantation. Data analysis displayed significant return of sheep's consciousness at $24(p=0.018), 48(p=0.017), 72(p=0.013)$ and 168 times ( $p=0.005)$ compared to the control group.

It should be noted that in the control group, clinical symptoms were abnormal until the end of the study and never returned to the state before inflammation (time of $-24 \mathrm{~h}$ ).

\section{MSCs: Balancing arterial blood gases and electrolytes}

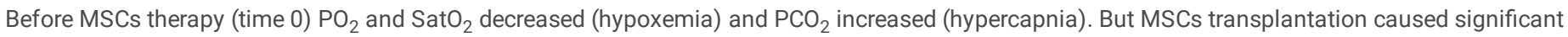
increase in $\mathrm{PO}_{2}$ and $\mathrm{SatO}_{2}$ at 24, 48, 72 and 168 hours in both compared with time 0 and compared with control group ( $\left.\mathrm{p}<0.05\right)$. Also, cell therapy caused significant decrease in $\mathrm{PCO}_{2}$ at 24, 48 and $72 \mathrm{~h}$ compared with time 0 and in compared with control group ( $\mathrm{p} \leq 0.05$ ) (Figure 2). This is while in the control group hypoxemia and hypercapnia continued until the end of the study. Additionally, respiratory acidosis was observed in both groups at time of 3 and $6 \mathrm{~h}$ because $\mathrm{PCO}_{2}$ increase and decrease in $\mathrm{pH}$. Analysis of $\mathrm{pH}$ value in the MSCs therapy group displayed significant difference at $48 \mathrm{~h}(\mathrm{p}=0.037)$ and $72 \mathrm{~h}$ $(p=0.044)$ compared to the PBS group. Survey of bicarbonate, anion gap and base excess data and the information of electrolytes including $\mathrm{Na}$, $\mathrm{K}$ and $\mathrm{Cl}$ indicated no remarkable difference in and between the two groups (Table 3).

\begin{tabular}{|c|c|c|c|c|c|c|c|c|c|}
\hline \multirow[t]{2}{*}{ Electrolytes } & Times (h) & -24 & 0 & 3 & 6 & 12 & 24 & 48 & \\
\hline & $\begin{array}{l}\text { Group } \\
\text { Treatment }\end{array}$ & $145.40 \pm 2.19$ & $150.80 \pm 3.63$ & $150.60 \pm 3.43$ & $148.80 \pm 3.70$ & $147.60 \pm 2.88$ & $147.80 \pm 2.77$ & $147.80 \pm 3.19$ & 1 \\
\hline $\mathrm{Na}(\mathrm{mmol} / \mathrm{l})$ & Control & $145.60 \pm 1.67$ & $150.60 \pm 3.57$ & $149.40 \pm 4.72$ & $148 \pm 3.53$ & $147 \pm 2.44$ & $147.80 \pm 5.40$ & $148.80 \pm 2.58$ & 14 \\
\hline \multirow[t]{2}{*}{$\mathrm{K}(\mathrm{mmol} / \mathrm{l})$} & Treatment & $4.44 \pm 0.35$ & $4.14 \pm 0.62$ & $4.10 \pm 0.27$ & $4.16 \pm 0.65$ & $4.14 \pm 0.62$ & $4.20 \pm 0.43$ & $4.26 \pm 0.70$ & 4.2 \\
\hline & Control & $4.52 \pm 0.38$ & $4.18 \pm 0.45$ & $4.14 \pm 0.59$ & $4.12 \pm 0.32$ & $4.16 \pm .30$ & $4.14 \pm .43$ & $4.18 \pm .56$ & 4. \\
\hline \multirow[t]{2}{*}{$\mathrm{Cl}(\mathrm{mmol} / \mathrm{l})$} & Treatment & $99.80 \pm 4.49$ & $106.80 \pm 7.79$ & $108 \pm 10.44$ & $108 \pm 6.12$ & $109 \pm 8.71$ & $108.20 \pm 7.49$ & $106.60 \pm 9.91$ & 10 \\
\hline & Control & $100 \pm 3.16$ & $106 \pm 4.69$ & $107.60 \pm 6.22$ & $108.80 \pm 3.70$ & $111.40 \pm 4.97$ & $111.20 \pm 4.54$ & $110.60 \pm 6.98$ & 109 \\
\hline \multirow[t]{2}{*}{ AnionGap (mmol/l) } & Treatment & $15.72 \pm 2.20$ & $16.84 \pm 2.06$ & $17.12 \pm 1.56$ & $17.20 \pm 2.46$ & $17.30 \pm 2.25$ & $17.18 \pm 1.21$ & $16.84 \pm 3.83$ & $16 . !$ \\
\hline & Control & $15.88 \pm 1.49$ & $16.94 \pm 1.23$ & $17.28 \pm 1.94$ & $17.44 \pm 0.70$ & $17.68 \pm 1.16$ & $17.62 \pm 1.77$ & $17.52 \pm 2.48$ & $17 .:$ \\
\hline
\end{tabular}

\section{MSCs: adjustment of cells of blood and BAL}

One day after inflammation (time 0), a significant increase observed in the absolute number of WBC (leukocytosis) and segmental and band neutrophils in the sheep (Figure 3). BM-MSCs transplantation in the treatment group reduced the number of WBCs. It was significant at hours of $24(p=0.043), 48(p=0.041), 72$ 
$(\mathrm{p}=0.037)$, and $168(\mathrm{p}=0.011)$ compared with time zero (before transplantation). In addition, comparison to the control group indicated significant difference at $24,48,72$ and 168 times ( $p$ value was $0.000,0.012,0.003$ and 0.038 , respectively).

In the control group, leukocytosis continued until the end of the study. MSCs decreased the number of segmental neutrophils, so that neutrophils resolved and returned to normal number after one week of transplantation. Statistical analysis revealed significant relationship at times of $24(p=0.033), 48(p=0.024), 72$ $(p=0.029)$, and $168(p=0.020)$ compared with time 0 and it was significant at $24(p=0.016), 48(p=0.031), 72(p=0.002)$, and 168 hours $(p=0.047)$ compared with the control group.

In addition, MSCs decreased the band neutrophils number and comparison to the PBS group displayed the significant difference at $24,48,72$, and 168 times ( $p$ value was $0.039,0.034,0.034$ and 0.028 , respectively). At time of inflammation, lymphocytes number increased but MSCs caused them to decline and their number returned to the base level at $168 \mathrm{~h}$. Statistical analysis displayed significant difference at $24 \mathrm{~h}(\mathrm{p}=0.012)$ compared to the control group only (Figure

3). Survey of the number of monocytes showed that, in the control group there is monocytosis while in the treatment group the absolute number of monocytes remained constant during the study. Comparison the treatment group with the control group revealed the significant difference at $72(p=0.040)$ and 168 hours $(p=0.034)$.

In addition, there was a significant difference in RBC at $24 \mathrm{~h}(\mathrm{p}=0.044), \mathrm{HGB}$ at times of $24(\mathrm{p}=0.034)$ and $48(\mathrm{p}=0.026)$ and HCT at 24 hour $(\mathrm{p}=0.044)$ in the cell receiver group in comparison with the PBS receiver group. But, there was no significant difference in the PLT count in or between the groups during the study (Figure 4).

The BAL results displayed that the cell count, macrophages and neutrophils were significantly increased in time of inflammation (Figure 5). Autologous transplantation of BM-MSCs modulated the inflammatory cells amount and the total cell count decline was significant at hours of $12,24,48,72$, and 168 compared with zero time ( $p$ value was $0.016,0.032,0.017,0.032$ and 0.023 , respectively) and at times of $24,48,72$ and $168 \mathrm{~h}$ in comparison to the control group ( $p$ value was $0.047,0.044,0.043$ and 0.043 , respectively). In addition, stem/stromal cells reduced alveolar macrophages number from $24 \mathrm{~h}$ to next while in the control group the number of macrophages did not decrease. Statistical analysis demonstrated the effectiveness of MSCs at 24 ( $p=0.012), 48$ ( $p=0.015)$ and 168 hours $(p=0.031)$ in comparison to the PBS group.

In addition, transplantation of BM-MSCs decreased the neutrophils number at $12(p=0.003), 24(p=0.007), 48(p=0.001), 72(p=0.001)$, and 168 times $(p=0.001)$ in comparison with before transplantation (time 0$)$ and at hours of $24(p=0.025), 48(p=0.034), 72(p=0.023)$ and $168(p=0.026)$ compared with the control group (Figure 5).

\section{MSCs: reconstruct and maintain the integrity of the capillary-alveolar membrane}

In ARDS model (time 0) the total protein amount, concentration of Ig M/total protein and albumin/total protein increased significantly in BAL which represents an increase in pulmonary vascular permeability. But MSCs therapy reduced their amount in BAL, so that reducing the amount of total protein was significant at times of 48,72 and 168 both compared with before transplantation (time 0$)(p<0.05)$ and compared with the control group ( $<<0.05)$. Additionally, decreasing Ig M/total protein concentration and albumin/total protein concentration were significant at hours of 72 and 168 both in comparison with before transplantation (time zero) $(p<0.05)$ and in compared with the control group $(p<0.05)$ (Figure 6$)$. These results indicated BM-MSCs reduce fluid leakage into the alveoli by restoring and maintaining the capillary-alveolar membrane integrity.

\section{MSCs: Decline in pro-inflammatory cytokines and enhancement of anti-inflammatory cytokine}

Twenty-four hours after inflammation (time 0), concentrations of proinflammatory cytokines (TNF-a/total protein and IL-6/total protein) increased and concentration of antiinflammatory cytokine (IL-10/total protein) decreased in BAL. But, stem cell therapy in the treatment group reduced TNF-a/total protein concentrations at 24,48 and 72 hours in comparison with time of zero ( $p$ value was $0.025,0.004$ and 0.009 , respectively) and at 24,48 and 168 times in comparison to the PBS reeiver group ( $p$ value was $0.042,0.043$ and 0.035 , respectively). In addition, in the treatment group concentration of IL-6/total protein decreased at hours of $24(p=0.001), 48(p=0.033), 72(p=0.001)$ and $168(p=0.038)$ in comparison with time of inflammation and at $24(p=0.047), 48$ $(p=0.034), 72(p=0.039)$ and 168 times $(p=0.034)$ compared with the group of control. Transplantation of BM-MSCs increased IL-10/total protein concentrations at 24,48 and 168 hours in comparison with zero time ( $p$ value was $0.040,0.015$ and 0.021 , respectively) and at times of 24 ( $p=0.041$ ) and 48 $(p=0.027)$ in comparison to the PBS group (Figure 7).

\section{Discussion}

ARDS is a relatively prevalent clinical syndrome, which impose major losses including physical and psychological complications and death. MSCs have proper effects in reducing acute pulmonary inflammation induced with LPS ${ }^{7,13,14}$. The results of this study revealed the BM-MSCs transplantation in sheep ARDS model led to clinical symptoms recovery, reconstruction of the capillary-alveolar membrane, balancing arterial blood gases and cytokines and adjustment of blood and BAL cells.

So far, few studies have been done on the large animal models such as sheep ${ }^{15}$ but similar to the present study, there is no the report that cells transplantation is autologous. Pre-clinical studies in a large animal model have better efficacy than laboratory animals such as small rodents ${ }^{16}$. Some researchers have stated that, with regards to the characteristics of a large animal model (such as sheep), including gas exchange, hemodynamic monitoring, and the acceptance of positive pressure ventilation, the same patients with ARDS model can use this animal model to compare with ARDS human model, as many of the features of which cannot be easily created in a laboratory animal model ${ }^{15}$. 
Our results were consistent with Mauricio et al.'s, study. They showed that the allogenic transplantation of human BM-MSCs administrated intratracheally reduced the intensity of inflammation and increased hemodynamic factors in the experimental ARDS model with LPS of E.coli in sheep ${ }^{17}$. In another study, researchers indicated that the intravenous allogenic transplantation of human BM-MSCs in ARDS sheep decreased the severity of inflammation and HR compared to the control group after $24 \mathrm{~h}$, significantly ${ }^{15}$. These results matched with the results of the present study, which demonstrated significant decline in $\mathrm{HR}$ at 24, 48 and $72 \mathrm{~h}$ after transplantation. Increasing blood neutrophils due to their role in processes of chemotaxis, opsonization and phagocytosis ${ }^{18}$ at zero time is a characteristic of leukogram inflammation and early stages of the disease. The significant decrease in the amount of inflammatory cells (WBC, neutrophils, lymphocytes and monocytes) has been reported following cell therapy in model of lung inflammation with endotoxin in mice ${ }^{19}$, which is consistent with the present study. Erythrocytosis, polycythemia and increase of hemoglobin in the present findings may have occurred following the reduction of blood plasma and dehydration of the animal or to compensate the oxygen deficiency of the body due to poor functioning of the heart or lung.

Most studies believe that direct damage to cytokines in inflammatory cascades is involved in pulmonary injury (Y. Li, et al., 2016). In this study, with assessment of cellular content and concentration of cytokines in BAL, the MSC treatment performance was evaluated. In the early stages of ARDS, most of the lung cellular secretions are neutrophils ${ }^{20}$. The various studies have shown that MSC can reduce the BAL neutrophils and it is clear that MSCs inhibit the activation and proliferation of immune cells by secreting a deterrent agent (Johnson et al., 2008; Meyer, 2007). Many of the current trends in the use of stem cells depend on the effects of soluble agents in these cells. The ability of these cells to secrete different paracrine agents, such as growth factors, endothelial and epithelial permeability regulators, anti-inflammatory cytokines and antimicrobial peptides, can be potentially useful in the treatment of ARDS underlying disorders, including capacity impairment, endothelial permeability change, dysregulated inflammation, and infection ${ }^{6,16}$.

BAL assessment after autologous transplantation of MSCs in this study displayed decrease of the total inflammatory cells, neutrophils and macrophages, proinflammatory cytokines (IL-6 and TNF-a) and the amount of total protein, Ig M and albumin and increase of anti-inflammatory cytokine (IL-10),these results were consistent with the study's results of Mei in mice ${ }^{13}$. Mei et al., induced pulmonary inflammation with LPS in mice intratracheally and administered MSCs intravenously after 30 minutes. The results of the BAL evaluation showed a significant decrease in the cell count, neutrophils and proteins ${ }^{13}$. MSCs, through angiopoietin I secretion and keratinocyte growth factor, induce restoration of alveolar vascular endothelial, and prevent leakage of protein-rich fluids as well as inflammatory cells, which leads to reduced alveolar edema ${ }^{13}$.

Xu et al., indicated intravenous transplantation of MSCs $1 \mathrm{~h}$ after intraperitoneal injection of LPS cause reduction of lung inflammation and edema. So that, the inflammatory cells numbers were decreased both as systematic and as local and serum concentration of IFN- $\gamma$, IL- $1 \beta$ and MIP, and neutrophils amount in histopathology also declined ${ }^{21}$. The use of MSCs in the treatment of acute pulmonary inflammation caused by the influenza virus ${ }^{6}, E$. coli bacteria ${ }^{22}$ and LPS of $E$. coli $^{23}$ in mice like our report represented that the amount of pro-inflammatory cytokines decreased in BAL and a corresponding increase was observed in the amount of anti-inflammatory cytokines. In another study, Hao et al., showed both human BM-MSCs and human ES-MSCs cause significant reduction of $E$. coli endotoxin-induced inflammation in mice but ES-MSCs did not demonstrate any useful effect on reducing edema of lung and permeability of the pulmonary vasculature compared with BM-MSCs. They stated that different MSCs tissues do not have the same behavior ${ }^{19}$.

Researchers represented menstrual blood-derived MSCs help to improve the permeability of the lung capillaries and reduce tissue damage by inhibiting IL-1 and increasing IL-10 in BAL and enhancing the expression of nuclear antigen (PCNA) and reducing the expression of caspase $3{ }^{24}$.

UC-MSCs have been able to inhibit the inflammatory response of macrophages in acute pulmonary inflammation caused by LPS in mice. After UC-MSC transplantation, pathological lesions reduced and inflammatory response including lung myeloperoxidase activity, protein concentration, neutrophils count, and expression of various inflammatory cytokines decreased in BAL at $72 \mathrm{~h}$ after transplantation ${ }^{25}$. The therapeutic capacity of UC-MSCs is primarily due to the secretion of paracrine, in particular prostaglandin (PGE2), and contributes to the improvement of lung damage by factors such as GM-CSF, IL-6 and IL-13 25

The transplantation of MSCs produces a systemic inflammatory response within a few hours. The results obtained from lavage and plasma studies indicate that cell therapy has a positive effect on the immune system. The systemic nature of these responses shows that MSCs in the lungs after transplantation can spread their effects to other parts of the body, and this may be the basis of the MSC therapeutic mechanism. Pro-inflammatory cytokines are a facilitator of the innate immune system and are mainly produced by macrophages and neutrophils and are called internal fever. These factors cause acute phase responses and production of acute phase proteins in the liver and inflammatory cells are activated. Cell therapy in the rabbit pulmonary inflammation caused a decrease in the pro-inflammatory cytokines and thereby improved hyperthermia ${ }^{7,26}$, similar to the results of this study.

The experimental inflammation induces an increase in alveolar macrophage cells. The accumulation of macrophages in the repair position leads to the secretion of various factors and cytokines. Leukocytes migration is largely driven by chemokines, and mutual communication between the early response of cytokines, sticky molecules and chemokines, coordinates neutrophils absorption into the lung. Active neutrophils and macrophages produce free oxygen radicals that play an important role in inflammatory pathways and lead to cellular damage in ARDS patients ${ }^{5}$. But stem cells transplantation cause a increases in the phagocytosis potential of macrophages and antimicrobial peptides in alveoli ${ }^{6}$. These represent the function of MSCs to improve the immune system and despite the cells transplantation in the lung, this therapeutic approach has remarkable systemic effects. On the other hand, these effects occur through a small number of MSCs, which are able to escape from the removal process and migrate to damaged sites ${ }^{27}$.

The study of the effect of human MSCs on the reduction of acute pulmonary damage induced by intravenous olycic acid in the pig showed that increasing IL8 in ARDS acts as a chemokine for neutrophil and has a close relationship with the intensity and duration of ARDS, and a significant relationship between neutrophils and concentration of IL8 was found but there was a significant reduction in NF-KB inflammatory factor transcription ${ }^{16}$. Also, the effect of human MSCs transplantation as intrapulmonary in sheep model of ARDS with intravenous endotoxin revealed the number of blood neutrophils and plasma level of 
IL-8 returned to before inflammation level while in the control group they did not return to normal until the end of the study. Total cells count cell, neutrophils and lymphocytes in BAL were fixed during the study, however, pulmonary edema was significantly eliminated after transplantation of MSCs ${ }^{17}$. Asmussen et al., did not observe significant change in amount of BAL neutrophils after MSCs intravenous therapy in model of acute lung inflammation in sheep but pulmonary edema was reduced.

Mokhber Dezfouli et al., demonstrated BM-MSCs intrapulmonary transplantation in ARDS rabbit model with E. coli LPS causes significant decline in total cell count, neutrophils, macrophages and pro-inflammation cytokines (IL- 6 and TNF-a) and a significant increase in cytokines inflammation (IL-10) in plasma and $\mathrm{BAL}$ and the rate of all of them returned to before inflammation levels. But in the control group these did not return to normal until the end of the study.

The results of the analysis of blood gases have an important role in the diagnosis and management of pulmonary capacity, the status of oxygenation and the balance of acid and base. Low levels of oxygen and high levels of carbon dioxide in the blood can occur due to decreasing gas exchange, which results from severe inflammation and obstruction of airway ${ }^{28}$. This condition occurred after the onset of inflammation in the present study. Cell therapy was able to improve arterial blood oxygen level by mediators and reducing inflammation. Stem cells transplantation induced a similar effect to bronchodilator drugs and made a significant increase in PO2 and SatO2 and a significant decrease in PCO2. These results matched with our previous study in ARDS rabbits model ${ }^{7}$. Also, researchers demonstrated human MSCs in ARDS of sheep cause increase in PO2 and decrease in PCO2 ${ }^{15,17}$. In cell therapy of influenza-induced pulmonary inflammation in mice, blood gas analysis has shown improvement in hypoxemia, which is consistent with the current study data ${ }^{6}$. Zhou et al., investigated the effects of mesenchymal cells isolated from bone marrow in pulmonary injury caused by aspiration of mouse gut contents. In this study, GFPpositive cells were transplanted by tail vein. Increasing partial pressure of arterial oxygen, decreasing protein levels and the total cells and neutrophils in BAL, decreasing TNF and cytokines caused by neutrophil activity and reduction of alveolar edema and the lung inflammation in histopathology are consistent with the current study ${ }^{25}$. In this study, there was no change in the acidity with metabolic origin. Hypoventilation, as it happened, at the time of zero to $6 \mathrm{~h}$ after inflammation resulted in accumulation of $\mathrm{CO}_{2}$ in the blood and reduction of $\mathrm{pH}$ (respiratory acidosis). Following cell transplantation, deep and fast breathing (hyperventilation) increased the removal of $\mathrm{CO}_{2}$ and resulted in an increase in $\mathrm{pH}$.

\section{Conclusions}

In general, the results of the present study showed that the BM-MSCs have an important role in the reconstruction of inflammatory lesions following intratracheal administration of LPS E. coli in sheep lung. These cells prevented the progression of inflammation and improved the clinical symptoms and local and systemic inflammatory factors. Therefore, the results of this study, along with further surveys can be used in the future for the treatment of ARDS in human.

\section{Abbreviations}

ARDS: Acute Respiratory Distress Syndrome

MSCs: Mesanchymal Stem/Stromal Cells

BM-MSC: Bone Marrow Mesanchymal Stem/Stromal Cells

LPS: Lypopolysacarid

PBS: Phosphate Buffer Solution

BM: Bone Marrow

IM: Intramuscular

GMP: Good Mmanufacturing Practice

DMEM-HG: Dulbecco's Modified Eagle Medium-High Glucose

FBS: Fetal Bovine Serum

CD45: Cluster of Differentiation 45

BAL: Bronchoalveolar Lavage

HR: Heart Rate

RR: Respiratory Rate

RT: Rectal Temperature

TNF-a: Tumor Necrosis Factor-a

IL-6: Interleukin 6 
ELISA: Enzyme-linked immunosorbent assay

WBC: White Blood Cell

$\mathrm{PO}_{2}$ : Partial Pressure of Oxygen

$\mathrm{PCO}_{2}:$ Partial Pressure of Carbon dioxide

RBC: Red Blood Cell

HGB: Whole Blood Haemoglobin Concentration

HCT: Hematocrit

PLT: Platelet

$\mathrm{SatO}_{2}: 02$ Saturation

ES-MSCs: Embryonic Stem cell-derived Mesanchymal Stem/Stromal Cells

UC-MSCs: Umbilical Cord-derived Mesanchymal Stem/Stromal Cells

HCO3: Bicarbonate

TCO2: Total CO2

\section{Declarations}

\section{Ethics approval and consent to participate}

All protocols of animals and experiments were checked and appropriated by the Animal Research Ethical Committee of University of Tehran (Tehran, Iran).

\section{Consent for publication}

Not applicable.

\section{Availability of data and material}

Authors want to share their data.

\section{Funding}

No funding was received.

\section{Competing interests}

The authors declare that they have no competing interests.

\section{Authors' contributions}

Sirous Sadeghian Chaleshtori; The study design, performed the cellular experiments, and prepared the initial, revision and finalization manuscript.

Mohammad Reza Mokhber Dezfouli; The study design, commented on the data analysis and the revision and finalization of the manuscript.

Javad Abbasi; Performed clinical experiments, the molecular studies and prepared the initial manuscript.

Mohammad Mehdi Dehghan; Analysis and interpretation the data and finalization of the manuscript.

Massoumeh Jabbari Fakhr, Performed the cellular experiments, the molecular studies and prepared the initial manuscript.

Shokufeh Yadollahi; Performed the cellular and clinical experiments.

Mohammad Mehdi Mirabad; Performed the clinical experiments.

All authors read and approved the final manuscript.

\section{Acknowledgements}

Not applicable.

\section{References}


1. Matthay, M.A. \& Zemans, R.L. The Acute Respiratory Distress Syndrome: Pathogenesis and Treatment. Annual review of pathology 6, 147-163 (2011).

2. Gonzales, J.N., Lucas, R. \& Verin, A.D. The Acute Respiratory Distress Syndrome: Mechanisms and Perspective Therapeutic Approaches. LID - 1009 [pii]. Austin J Vasc Med (2016).

3. Mei, S.H., Dos Santos, C.C. \& Stewart, D.J. Advances in Stem Cell and Cell-Based Gene Therapy Approaches for Experimental Acute Lung Injury: A Review of Preclinical Studies. Human Gene Therapy (2016).

4. Cribbs, S.K., Matthay, M.A. \& Martin, G.S. Stem cells in sepsis and acute lung injury. Critical care medicine 38, $2379-2385$ (2010).

5. Dushianthan, A., Grocott, M.P.W., Postle, A.D. \& Cusack, R. Acute respiratory distress syndrome and acute lung injury. Postgraduate Medical Journal 87, 612 (2011).

6. Li, Y., et al. Mesenchymal stromal cell treatment prevents H9N2 avian influenza virus-induced acute lung injury in mice. Stem Cell Research \& Therapy 7, 159 (2016).

7. Mokhber Dezfouli, M.R., et al. Intrapulmonary autologous transplant of bone marrow-derived mesenchymal stromal cells improves lipopolysaccharideinduced acute respiratory distress syndrome in rabbit. Crit Care (2018).

8. Matute-Bello, G., Frevert, C.W. \& Martin, T.R. Animal models of acute lung injury. American journal of physiology. Lung cellular and molecular physiology 295, L379-L399 (2008).

9. Kamaruzaman, N.A., Kardia E Fau - Kamaldin, N.A., Kamaldin N' Fau - Latahir, A.Z., Latahir Az Fau - Yahaya, B.H. \& Yahaya, B.H. The rabbit as a model for studying lung disease and stem cell therapy. BioMed Research International (2013).

10. Ranieri Vm Fau - Rubenfeld, G.D., et al. Acute respiratory distress syndrome: the Berlin Definition. (2012).

11. Patterson, C. Veterinary Medicine: A Textbook of the Diseases of Cattle, Horses, Sheep, Pigs, and Goats, 11th edition, Volumes 1 and 2. Can Vet J 58, 1116-1116 (2017).

12. Mokhber Dezfouli, M.R., et al. Changes in clinical signs after treatment in calves with experimental colisepticemia with Escherichia coli. Journal of Veterinary Research 72, 63-71 (2017).

13. Mei, S.H.J., et al. Prevention of LPS-Induced Acute Lung Injury in Mice by Mesenchymal Stem Cells Overexpressing Angiopoietin 1. PLoS Medicine 4, e269 (2007).

14. Rojas, M., et al. Infusion of freshly isolated autologous bone marrow derived mononuclear cells prevents endotoxin-induced lung injury in an ex-vivo perfused swine model. Stem Cell Research \& Therapy 4, 26-26 (2013).

15. Asmussen, S., et al. Human mesenchymal stem cells reduce the severity of acute lung injury in a sheep model of bacterial pneumonia. Thorax (2014).

16. Moodley, Y., et al. Human mesenchymal stem cells attenuate early damage in a ventilated pig model of acute lung injury. Stem Cell Research (2016).

17. Rojas, M., et al. Human adult bone marrow-derived stem cells decrease severity of lipopolysaccharide-induced acute respiratory distress syndrome in sheep. Stem cell research \& therapy 5, 42-42 (2014).

18. Abraham, E. Neutrophils and acute lung injury. Crit Care Med (2003).

19. Hao, Q., et al. Study of Bone Marrow and Embryonic Stem Cell-Derived Human Mesenchymal Stem Cells for Treatment of Escherichia coli EndotoxinInduced Acute Lung Injury in Mice. Stem Cells Translational Medicine 4, 832-840 (2015).

20. Kinoshita, M., Ono S Fau - Mochizuki, H. \& Mochizuki, H. Neutrophils mediate acute lung injury in rabbits: role of neutrophil elastase. Eur Surg Res (2000).

21. Xu, Y.L., et al. Intravenous transplantation of mesenchymal stem cells attenuates oleic acid induced acute lung injury in rats. Chin Med $J$ (Engl)Chin Med $J$ (Engl) (2012).

22. Gupta, N., et al. Mesenchymal stem cells enhance survival and bacterial clearance in murine Escherichia coli pneumonia. Thorax. (2012).

23. Gupta, N., et al. Intrapulmonary delivery of bone marrow-derived mesenchymal stem cells improves survival and attenuates endotoxin-induced acute lung injury in mice. The Journal of Immunology (2007).

24. Xiang, B., et al. Transplantation of Menstrual Blood-Derived Mesenchymal Stem Cells Promotes the Repair of LPS-Induced Acute Lung Injury. LID - E689 [pii] LID - 10.3390/ijms18040689 [doi]. Int J Mol Sci (2017).

25. Zhu, H., et al. Therapeutic Effects of Human Umbilical Cord-Derived Mesenchymal Stem Cells in Acute Lung Injury Mice. Scientific Reports (2017).

26. Zhu, F., et al. [Effect of bone marrow-derived mesenchymal stem cells transplantation on the inflammatory response and lung injury in rabbit with inhalation injury]. Zhonghua Shao Shang Za Zhi (2010).

27. Abbasi, J., et al. Improvement of clinical signs in experimental model of Acute Respiratory Distress Syndrome (ARDS) in sheep following autograft of bone marrow-derived mesenchymal stem cells (BM-MSCs), (2018).

28. Sei, K., et al. Appropriate timing of blood sampling for blood gas analysis in the ventilated rabbit. J Surg Res (2016).

\section{Figures}




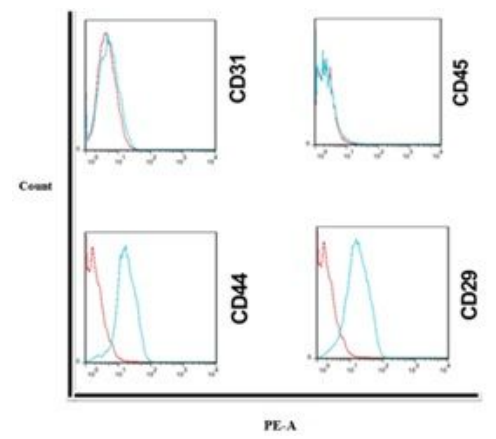

Figure 1

BM-MSCs analysis of flow cytometry. Flow cytometry analysis displayed surface antigen expression of CD44 (89\%) and CD29 (91\%) and were negative for CD45 and CD31.The blue graph demon־strated the surface antigens level and the red graph showed the control samples.

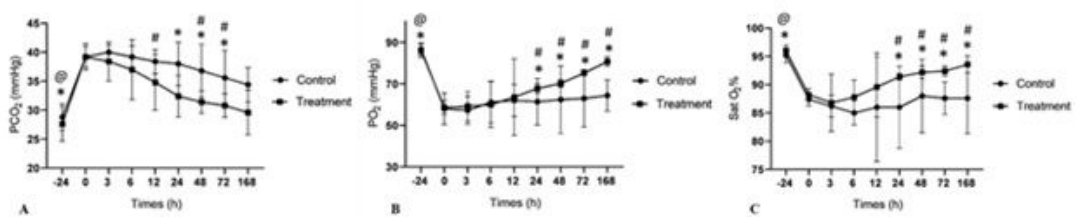

Figure 2

The sheep arterial blood gases pressure (mean \pm SD) in the treatment group (ARDS+BM-MSCs) and the control group (ARDS+PBS) in the period of study. (A) PCO2, (B) PO2, (C) SatO2.

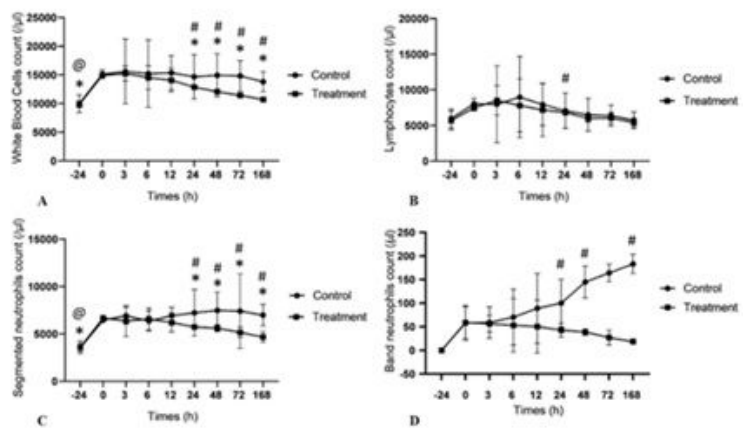

Figure 3

The sheep hematological factors (mean \pm SD) in the treatment group (ARDS+BM-MSCs) and the control group (ARDS+PBS) in the period of study. (A) WBCs, (B) lymphocytes, (C) Segmented neutrophils, (D) Band neutrophils.

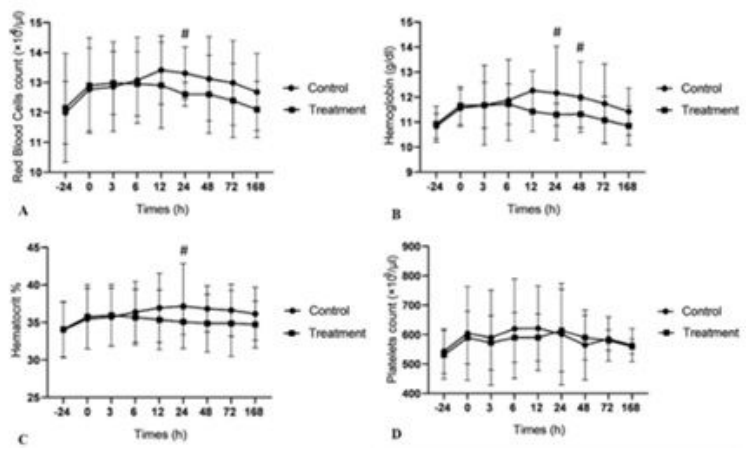

Figure 4

The sheep hematological factors (mean \pm SD) in the treatment group (ARDS+BM-MSCs) and the control group (ARDS+PBS) in the period of study. (A) RBCs, (B) HGB, (C) HCT, (D) PLT. 


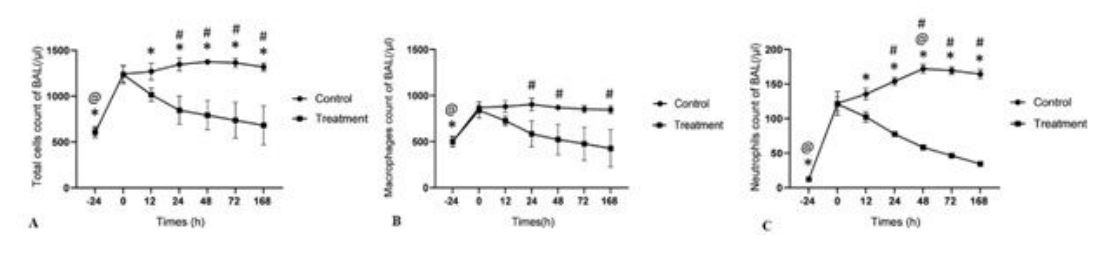

Figure 5

The sheep BAL cells amount (mean \pm SD) in the treatment group (ARDS+BM-MSCs) and the control group (ARDS+PBS) in the period of study. (A) Total cells, (B) Macrophage, (C) Neutrophil.

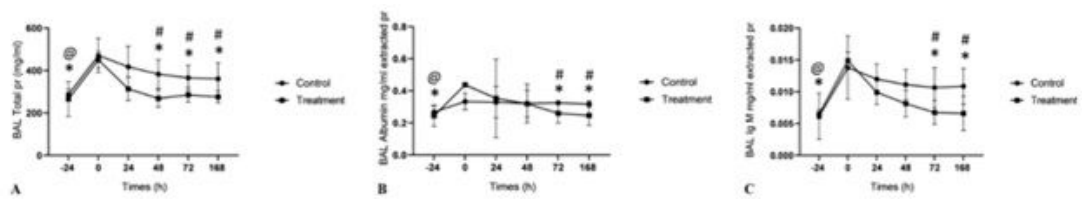

\section{Figure 6}

The sheep total protein amount (A), albumin/total protein concentration (B) and Ig M/total protein concentration (C) (mean \pm SD) in the treatment group (ARDS+BM-MSCs) and the control group (ARDS+PBS) in the period of study.
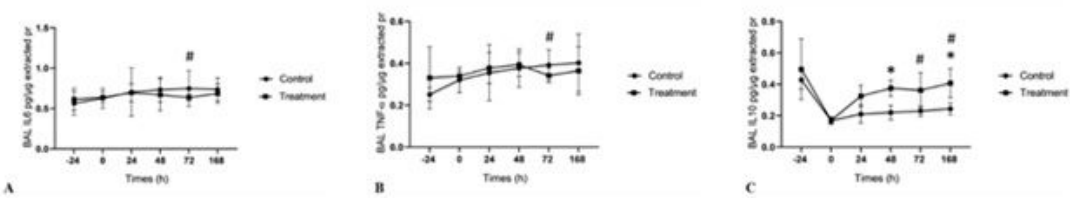

Figure 7

The sheep BAL cytokines/total protein amount (mean \pm SD) in the treatment group (ARDS+BM-MSCs) and the control group (ARDS+PBS) in the period of study. (A) IL-6/ total protein concentration, (B) TNF-a/total protein concentration, (c) IL-10/total protein concentration. Note: In the tables and figures, information are as mean $\pm S D\left(n=5\right.$ sheep/group). ${ }^{*} p<0.05$; The changes that are significantly in comparison with time 0 in the same group. \#p $<0.05$; The changes that are significantly in comparison to the group of control (ARDS+PBS) at the same time. 